\title{
Novel Voltage Equalizers for Secondary Batteries including EDLCs using CW circuit
}

\author{
Mikio Yasubayashi $^{\mathrm{a}}, \quad$ Keiju Matsui ${ }^{\mathrm{b}, *}$, Eiji Oishi $^{\mathrm{b}}$, Masayoshi Umeno ${ }^{\mathrm{a}}$ \\ Hideo Uchida ${ }^{\text {a }}$, Yasutaka Kawata ${ }^{\text {a }}$, Masaru Hasegawa ${ }^{a}$ \\ ${ }^{a}$ Chubu University, Kasugai 487-8501, Japan \\ ${ }^{\mathrm{b}}$ Minna-denryoku, Inc., Setagaya Monozukuri Gakko, room 210, Setagaya154-0001, Japan \\ *Corresponding Author:
}

\begin{abstract}
Various secondary batteries including EDLCs are widely used in various electric vehicles, etc. Among them, EDLCs offer high energy density and long life span, so various applications may be anticipated in the realm of those energy storage devices. However, since the voltage limit is low of the devices, it is necessary to connect them in series or parallel. In addition, it is required that they be used in the region of their critical voltage limit or capacity limit. In order to apply them efficiently, the devices should be used with balanced voltage. In this paper, some novel voltage balancers are presented, employing a $\mathrm{CW}$ (Cockcroft-Walton) circuit as a basic construction. Characteristics of proposed circuits are analyzed and improved especially about circuit construction strategies.
\end{abstract}

Keywords: Secondary battery1, EDLC2; voltage equalizer3, Cockcroft-Walton circuit4, buck-boost chopper 5

\section{Introduction}

Secondary Batteries as energy storage devices have been examined and reported. Among them, EDLCs (Electric Double Layer Capacitors), which are often called supercapacitors, can offer high energy storage performance in terms of surge power, efficiency, cold temperature operation and large number of energy cycles ${ }^{(1)}$.

For these reasons, in power compensating equipments for voltage fluctuations or instantaneous voltage drop in the power systems, EDLCs are expected to be applied as energy storage equipments. Additionally, in various vehicles, such as electric cars and trains, these applications have just been introduced. In such EDLCs, however, as voltage limit of devices is low, it is necessary to connect them in series or parallel configurations, and to use them in the vicinity of their voltage limit. Consequently, in order to use efficiently, these devices must be used in well balanced manner. Amongst various voltage balancing techniques, a balancing method using resistors can be applied as the most fundamental, simple and effective solution ${ }^{(2)}$. When considering the power losses, however, such methods have restricted application in practice. Another method, using Zener diodes, has been discussed and evaluated ${ }^{(1,3)}$. In considering the energy consumption of such Zener diodes, the power capacity of the system may be limited. Methods employing chopper circuits have also been proposed ${ }^{(3,4)}$, but the number of switching devices and their accompanied control circuitry is increased, leading to the high cost of such systems. Other original strategies have been presented and components ${ }^{(5)}$. Though the inverter circuit is complicated and charging operations are needed, such methods are suited to the required increased capacities. The most orthodox method is thought to be the forward converter method, using transformers, which accompany each EDLC, and charge and discharge through their primary and secondary windings ${ }^{(6,7)}$. Similarly to solution ${ }^{(3)}$, their controls may be complicated by many devices like transformers. Although such devices are necessary, however, their size is very small. Thus, this technique is expected to be widely used in extensive applications like the electric vehicles $^{(8,9)}$.

Considering the various types of EDLCs voltage balancers, perfect or even adequate solutions have not obtained. However, in the future, various other methods will be studied and proposed. In the light of the above research into voltage balancers for EDLCs, we had initially studied reference ${ }^{(10,11)}$, and derived novel methods which was examined and discussed ${ }^{(10-12)}$. An alternative approach to 
voltage balancing was presented, employing a Cockcroft-Walton circuit (CW circuit), which was invented long ago ${ }^{(13)}$ for high dc voltage generation and employs numbers of capacitors and diodes. By means of this $\mathrm{CW}$ circuit, EDLCs having different capacitances were made to provide identical voltage. Voltage balancing is achieved with a high frequency power supply or buck-boost chopper. Their analyzed results and the mechanism are presented and discussed. In this paper, these results are applied to balance the small voltages of EDLCs. Another splendid equalizer is also proposed ${ }^{(15,16)}$, in which the cells are controlled by means of a string of reference capacitors and double groups of many switches. The balancing operation is a little analogous to the $\mathrm{CW}$ circuit, so its principle is interesting. The circuit and its operation, however, is a little complicated, many switches should be provided and yet the operational principle is entirely different. Under such background, in March, 2008, a novel voltage equalizer using CW circuit had been proposed ${ }^{(14)}$ firstly in the world by one of the authors. Various further versions and their results are to be presented and discussed including experiments.

\section{Fundamental Circuit Configuration}

\subsection{Operation Analysis in AC Power supply}

Fig.1 shows the proposed circuit for the balanced charging of EDLCs, using CW (Cockcroft-Walton) circuit. $\mathrm{C} 1 *$ to $\mathrm{C}^{*}$, on the left hand side, indicate, for example, electrolytic capacitors, which have relatively uniform values and can be obtained at low cost. e is the ac power supply. The purpose is not to supply the output power to these, but to supply

relatively reduced power in order to balance the EDLC voltage on the right hand side. These EDLCs can be replaced by usual secondary batteries if it is necessary. In this paper, the subject of study is EDLCs. By means of adjusting the voltage or frequency, voltage equalizing conditions can be obtained. By an operation of e, EDLCs. can be held effectively in voltage equalization.

\section{Double Switches Method}

Fig.2 shows another version of voltage equalizer using modified CW circuit having double switches. As comparison to already reported buck-boost chopper [16], their main switches are removed, the inductors of chopper is replaced by diodes and double switches $S_{1}, S_{2}$ are equipped, which are turned on and off periodically. By means of this construction, simple equalizer can be obtained.

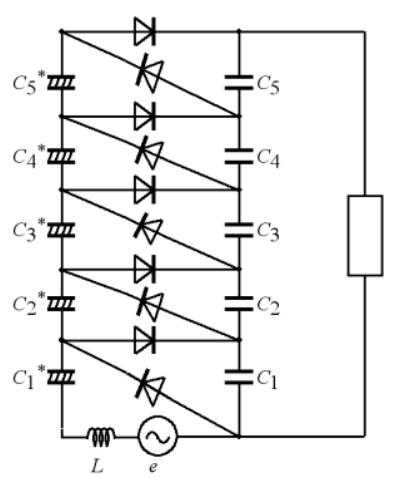

Fig.1. Basic equalizer with $\mathrm{CW}$ circuit.

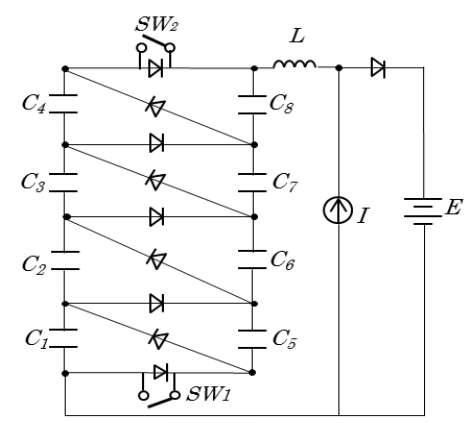

Fig.2. Double switches equalizer.

\subsection{Equalizing operation}

a) circuit operation at turned-off. The charging current is flowing through double paths root 1 and root 2 in Fig.3. $\mathrm{C}_{1}$ or $\mathrm{C}_{8}$ shares each current in parallel path whose voltage becomes about double compared to others.

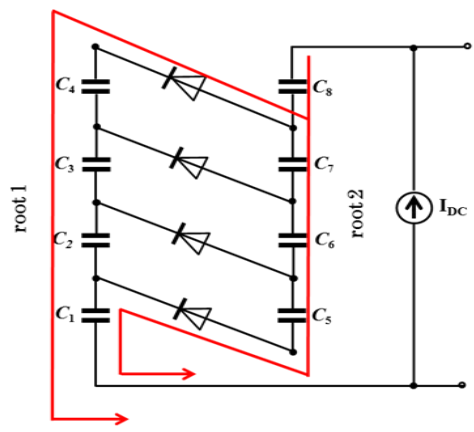

Fig.3. The circuit operation at switch turned-off.

b) Circuit operation at turned-on. Due to turned-off operation, for example, as $\mathrm{C}_{8}$ voltage is higher than $\mathrm{C}_{4}$ voltage, excess charge is delivered from $\mathrm{C}_{8}$ to $\mathrm{C}_{4}$ through root 3 as shown in Fig.4. Also in the bottom stage, the excess charge is delivered from $C_{1}$ to $C_{5}$ through root 4 in the similar reason.

\subsection{Voltage equalizing operation by simulation}

Also in the other stages, the excess charge of left hand 
side capacitor is delivered toward that opposite side capacitor at the right hand side. As such operation at turned-off, the excess charge of capacitor at the right hand side is delivered toward just upper side capacitor of the opposite side. From such operations, we might consider whether above switch $S_{1}$ is necessary or not. For turned-off period, however, the excess charge of $\mathrm{C}_{8}$ due to root 1 and root 2 in Fig. 3 must be delivered to $\mathrm{C}_{4}$. And also, the excess charge of $\mathrm{C}_{4}$ should be delivered toward $\mathrm{C}_{8}$ In such way, double switches are necessary for the transmittal operation of excess charge from the right hand side capacitor to just lower side capacitor in the opposite side of the left hand side. For above description, simultaneous operation of both switches was verified and discussed. The simultaneous operation of both ones is not always necessary. Even though with alternating switching, operation can be performed successfully, where one switch is open and other is closed, or vice versa. Even though the frequency is different each other, the voltage equalization can be obtained.

In Fig.5, region $\mathrm{A}$ is called constant current charging operation and $\mathrm{B}$ is constant voltage one. This figure shows equalization characteristic, where $\mathrm{C}_{1}$ to $\mathrm{C}_{8}=1 \mathrm{mF}$ except $\mathrm{C}_{5}$ $=0.6 \mathrm{mF}$ reduced by deterioration, and duty ratio $=20 \%, I_{D C}$ $=0.1 \mathrm{~A}, E=100 \mathrm{~V}$. For the first rising region shown by $\mathrm{A}$, the equalizing characteristic can be kept satisfactorily as shown, but for the region B shows little discrepancies in the curves,

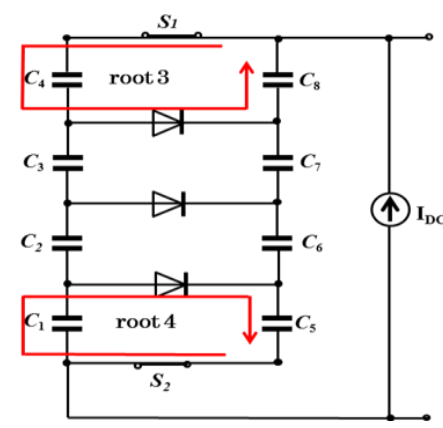

Fig.4. The circuit operation at switch turned-on.

which becomes slightly out of desired value of $25 \mathrm{~V}$. The reason can be explained as follows; in the region $\mathrm{A}$, compensating effect due to switching is more significant. Meanwhile, in the region $\mathrm{B}$, such effect due to switching is a little worse. In order to improve the equalization characteristic, the duty factor is increased to $30 \%$, where such result is shown in Fig.6. In the figure, by means of merely turned-on and off, satisfactory characteristic can be obtained, where every EDLC voltage is completely converging toward the desired value of $25 \mathrm{~V}$.
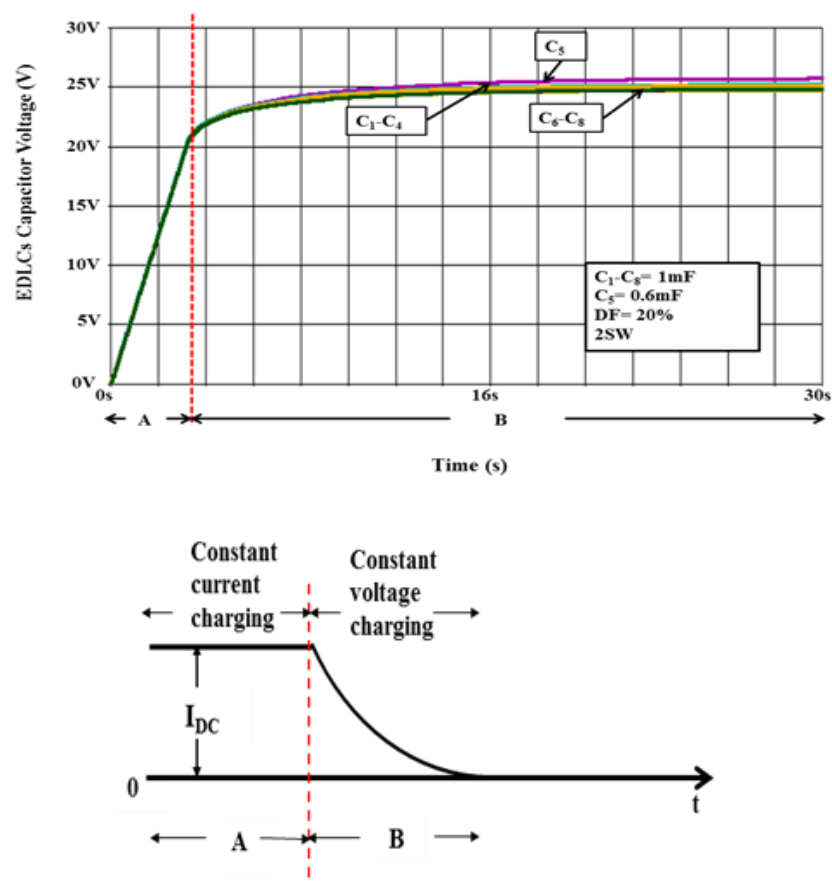

Fig.5. Equalizing characteristic with double switches.

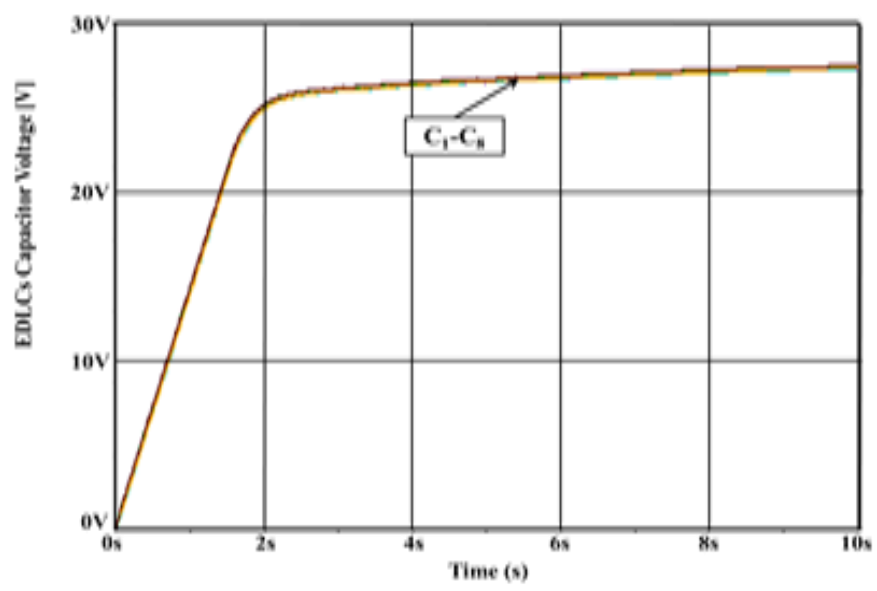

Fig.6. Satisfactory equalizing characteristic.

On the other hand, when one switch $S_{1}$ is removed from the equalizer, equalizing characteristic is lost, whose characteristic is shown in Fig.7. $\mathrm{C}_{8}$ voltage is increased about double, because current is flowing in parallel path through $\mathrm{C}_{8}$. For $\mathrm{C}_{1}$ voltage, however, by attached switch voltage is somewhat compensated.

\section{Single Switch Method}

Instead of double switches method, single switch method is proposed in this chapter. Double switches in Fig. 2 are taken the place by double inductors and added another switch as shown in Fig.8. By means of switching of $\mathrm{S}$, the voltage is applied across the terminal of equalizer. As a result, in the equalizer, 


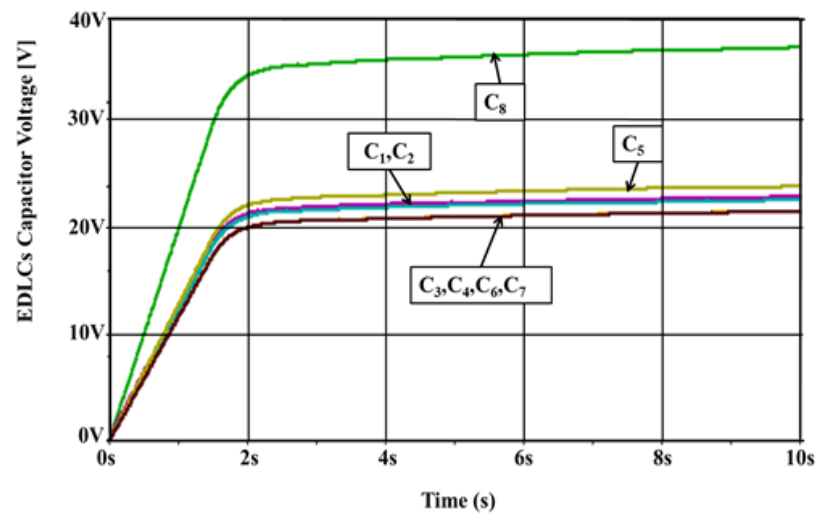

Fig.7. Unsuccessful equalizing characteristic without S1

\section{Single Switch Method}

Instead of double switches method, single switch method is proposed in this chapter. Double switches in Fig.2 are taken the place by double inductors and added another switch as shown in Fig.8. By means of switching of $\mathrm{S}$, the voltage is applied across the terminal of equalizer. As a result, in the equalizer,

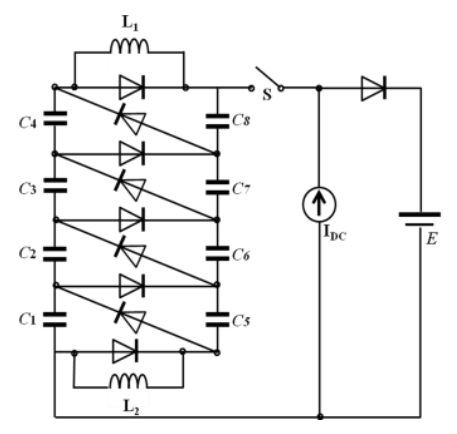

Fig.8. Single switch equalizer.

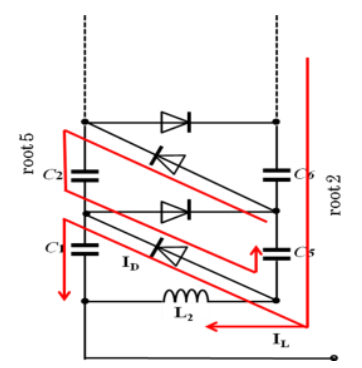

Fig.9. Operation circuit at turned-on.

current flow can be altered according to the switching state.

In Fig.9, when the switch is turned-on, the supply voltage is applied thr equalizer terminal. However, the current flows through inductor as IL in addition to ID, as shown. At the beginning, this current $\mathrm{IL}_{\mathrm{L}}$ is fairly reduced value because of adequate large inductance of L2. However, this current flows through $\mathrm{C}_{1}$ as ID. Because of this function, the current source type is preferable as the power supply. If the voltage of $\mathrm{C}_{5}$ is larger than $\mathrm{C}_{2}$ one, the current though root 5 as shown.

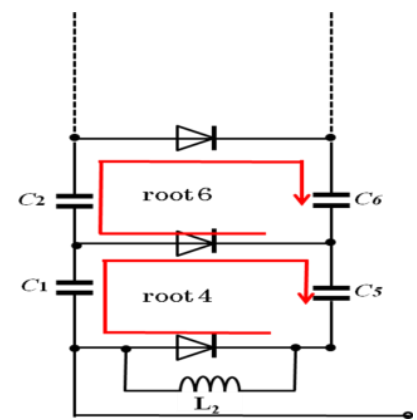

Fig.10. Operation circuit at turned-off.

In Fig. 10, the switch is turned-off, when the current flows through $\mathrm{L}_{2}$. As a result, The excess charge of $\mathrm{C}_{1}$ can be transmitted toward $\mathrm{C}_{5}$ side. By means of repetition of switching, both column voltages can be kept an identical value.

\section{Experiment and its Discussions}

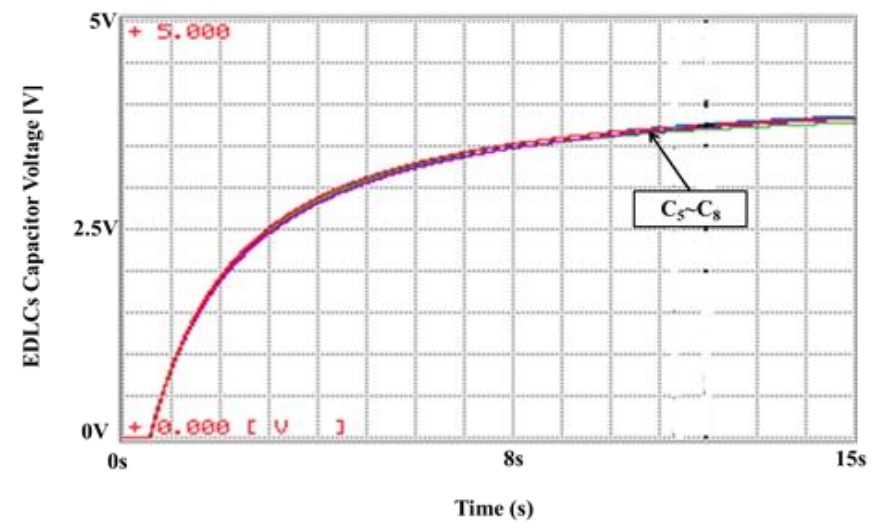

Fig.11. Equalizing characteristic at experiment.

Fig.11 shows voltage equalizing characteristic at trial experiment.The term "trial" means that instead of EDLC, electrolytic capacitors are used because sometimes unexpected excess charge was generated in a process of various experiments. Because of application of higher voltage against EDLC having lower voltage limit, electrolytic capacitor was chosen as experimental capacitor. External power supply is $12 \mathrm{~V}$ dc supply having series resistance of $100 \mathrm{ohm}$ which behaves like a current source. Each capacitance is $6.8 \mathrm{mF}$ except assumed deteriorated $\mathrm{C}_{5}$ of $3.4 \mathrm{mF}$. Switching frequency is $60 \mathrm{~Hz}$ in which both switch operate simultaneously. Every curve lies on single line. It can be seen that equalization characteristic is performed successfully. 


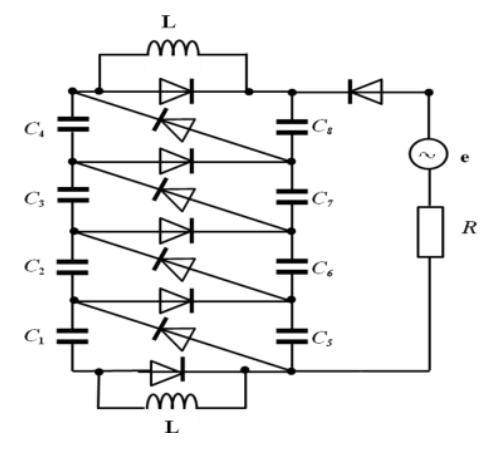

Fig.12. Single switch equalizer for experiment.

In the experiment of single switch equalizer, experimental circuit can be modified into simple configulation, whose circuit construction is shown in Fig.12. The half wave rectifier can be substituted for switching device.

\section{Conclusions}

A novel voltage equalizer using a CW circuit with switching has been proposed and discussed.

It is easy to apply, because of its simple and concise construction. The purpose of this system is not to obtain boosted power, but to obtain balanced voltages. Since such delivered power is not large, the ac power supply or switches are small in size.

The proposed circuit using a CW approach is compared with the forward converter method [9-10], which is called the conventional method in this paper and that application might be most orthodox way at this time.

i) The conventional method needs a transformer, even though the size is small because of high frequency used. Transformer and its design are complicated to every EDLC installation. The proposed method does not need particular design, and can be used as general-purpose.

ii) The proposed method needs a corresponding number of auxiliary capacitors. However, it may be possible that such capacitors can be replaced by EDLC, in which case, the system storage capacity could be significntly increased.

iii) The proposed method has a little deteriorated characteristic, in terms of circuit response, because the command is gradually delivered from the bottom to the top side. The conventional method is advantageous in relation to response. However, the aging time lapse is long, so quick response may be unnecessary for voltage balancer of EDLCs.

iv) For a case of using EDLC only having double switches or single one, circuit becomes in much simple construction, but equalizing operation can be obtained only at charging period. During discharging period such equalizing characteristic could not obtained unfortunately.
In this period, however, such feature could be accepted because a fatal over voltage would not be generated

\section{Acknowledgment}

This research is mostly supported by a grant of the NEDO (New Energy and Industry Technology Development Organization). We would like to express our appreciation to who it may concern about this project.

\section{References}

(1) Michio Okamura : "Electric Double Layer Capacitors and its Energy Storage Systems", 3rd Edition, Nikkan Kogyo Shinbun-sha, 2005

(2) Akitoshi Minemura, Masahiro Yashiro, Yasuyoshi Kaneko, Shigeru Abe : "Equalization of the Voltages Using Passive Resistors for Electric Double Layer Capacitors", The 2007 National Convention Record of IEE Japan, no.4-018, 2007

(3) Philippe Barrade, Serge Pittet, and Alfer Rufer : "Energy storage system using a series connection of EDLCs, with an active device for equalizing the voltages" IPEC-Tokyo-2000, pp.1555-1560, 2000

(4) Alfer Rufer, and Pilippe Barrade, "A EDLC-Based Energy-Storage System for Elevators With Soft Commutated Interface", IEEE Transaction On Industry Applications, VOL.38, NO.5, SEPTEMBER/ OCTOBER 2002

(5) Takatsugu Kishi, Toshihisa Shimizu : "A Study of Voltage Balancer for Electric Double Layer Capacitors", Technical Meeting on Semicondector Power Converter SPC-04-37, 2004

(6) Kazuya Mori, Akio Hasebe, Kiko Tsuruga, Takahiko Itoh, Sumiko Seki : "Voltage Balancer for Electric Double Layer Capacitors", The 2001 National Convention Record of IEE Japan, no.4-207, 2001

(7) Eiji Sakai, Koosuke Harada, S.Muta, Kiyomi Yamasaki ; "Swiching Converters using Double-Layer Capacitors as Power Backup", The 19th International Telecommunication Energy Conference, Proceedings of IEEE-Intelec 1997, pp.611-616, Oct.,1997

(8) Nasser H. Kutkur, Deepak M. Divan and Donald W. Novotny : "Charge Equalization for Series Connected Battery Strings", IEEE Transaction on Industry Applications, vol.31, no.3, pp.562-568, May/June, 1995

(9) H.Sakamoto,K.Murata,E.Sakai,,K.Nishijima,K.Harada, S.Taniguchi, K.Yamasaki, G.Akiyoshi : "Voltage Balanced Charging of Series Connected Battery Cells", The 20th International Telecommunication Energy Conference, Proceedings of IEEE-Intelec 1998, pp.311-315, Oct.,1998

(10) Keiju Matsui, Hiroto Shimada, Masaru Hasegawa : "Novel Voltage Balancer for an Electric Double Layer Capacitor by using Forward Converter ",The 4th International Telecommunication Energy Special Conference, Vienna, Proceedings of IEEE-telescon 2009, II.3-1,pp.1-6, May, 2009 
(11) Kimihiro Nishijima, Hiroshi Sakamoto, Koosuke Harada : "Voltage Equalizing System for Series Connected Battery Cells", IEICE Trans. On B,vol.J84-B,no.9, pp.1701-1708, Sep. 2001

(12) Jonathan W. Kimball, B. T. Kuhn and P. T. Krein : "Increased Performance of Battery Packs by Active Equalization," IEEE Vehicle Power and Propulsion Conference, pp. 323-327, Sep. 2007.

(13) J. D. Cockcroft, E. T. S. Walton : "Further development on the method of obtaining high velocity positive ions", Proc. Royal Society London, UK, 1932.

(14) Keiju Matsui, Isamu Yamamoto, Masaru Hasegawa, Hiroto Shimada : " A Novel Voltage Balancer for EDLCs Using Cockcroft-Walton Circuit”, 2008 National Convention Record IEE Japan vol.4, 4-138, pp.230-231, 2008 